\title{
Multiband Common Spatial Pattern based EEG Classification for Brain-Computer Interface
}

\author{
Md. Sujan Ali ${ }^{1}$, Mst. Jannatul Ferdous ${ }^{1}$ \\ ${ }^{I}$ Department of Computer Science and Engineering, JatiyaKabiKaziNazrul Islam University, Mymensingh, \\ Bangladesh
}

\begin{abstract}
Thispaper presents a novel method for electroencephalography (EEG) based motor imagery classification for brain computer interface (BCI) implementation using the potential features extracted bandspecific common spatial pattern (CSP). The recorded EEG signal is bandpass-filtered into multiple subbands to capture the related rhythmic components of brain signals. The CSP features are then extracted from each of these bands. The linear discriminant analysis (LDA) based classifier is subsequently used to classify the relevant subband of EEG using the features extracted by CSP. Then the highest discrimination score among all the subbands is used as the norm for overall EEG classification. The experimental results show that the proposed method yields comparatively superiorclassification performance compared to prevailing methods.
\end{abstract}

Keywords:brain computer interface (BCI), electroencephalography, common spatial pattern, filterbank.

\section{Introduction}

Brain-computer interface (BCI) is a communicating system between a brain and a device that enables signals from the brain to direct some external devices, such as a computer, wheelchairs [1], robotic arms, prostheses [2] etc. The interface translates human thoughts into command to control the external devices. The keytarget of the $\mathrm{BCI}$ is to restore or repair useful function to people disabled by neuromuscular disorders such as Amyotrophic Lateral Sclerosis (ALS), cerebral palsy, stroke, or spinal cord injury. Although people may become totally paralyzed through these types of disorders their minds are un-affected. Considering this issue brain computer interface translates human thoughts directly to the external world [3]. Electroencephalography (EEG) is a widely used non-invasive $\mathrm{BCI}$ due to its low expense and high-temporal resolution[4]. The feature extraction stage is responsible for forming discriminative set of features in the form of frequency patterns, temporal patterns, time-frequency patterns, autoregressive models, or spatial patterns for each task performed[5, $6,7]$. The features extracted are used to train a classifier to decode the users' intent and subsequently translate the features into a set of output commands for operating an external device.

The common spatial pattern (CSP) is an algorithm commonly used in BCI systems to preprocess the electroencephalogram (EEG) signals $[8,9,10]$.The algorithm finds optimal spatial filters that are functional in discriminating two classes of EEG signals in motor imagery based BCI. The effectiveness of the spatial filters depends on its subject specificfrequency band i.e. the performance varies with subjects as well as frequency bands.If the EEG signalsis unfiltered or have been filtered with badly chosen frequency rangethen the classification of that signals using CSP shows poor accuracies [11]. Consequently, subjectspecific frequency bands are generally used with the CSP algorithm [12].

To overcome the limitation of manually selecting the subject specific frequency bands for the CSP, the common spatio- spectral pattern (CSSP) algorithm has been proposed where simple filters are optimized together with the CSP algorithm [13]. The common sparse spectral-spatial pattern (CSSSP) algorithm improves the performance of CSSP algorithm. It allows concurrent optimization of an arbitrary finite impulse response (FIR) filter within CSP analysis [12]. Another approach called SPECtrally weighted common spatialpattern (SPEC-CSP) algorithm [14] optimizes the temporal filter in the frequency domain and after that the spatial filter in an iterative method [15]. However, due to the inherent nature of optimization problem, the solution of filter coefficients significantlydepends on the selection of initial parameters [11].

Sub-band CSP (SBCSP) method [11] was alternatively proposed and has been shown better classification accuracy compared against CSSP and CSSSP. In this method publicly available dataset from BCI competition III in 2005 has been used. As a substitute of temporal FIR filter within the CSP algorithm, SBCSP uses a filter bank that decomposes the EEG signals into sub-bands. The CSP algorithm is then employed on each of these sub-bands to obtain sub-band scores. To fuse the sub-band score two fusion methods namely recursive band elimination (RBE) and meta-classifier (MC) are used. An additional classifier is then usedto classify the fused sub-band scores. In [11] comparative study of using different sub-band score fusion techniques and classification algorithms are not available.The filter bankcommon spatial pattern (FBCSP) algorithm is proposed to classify EEG signals[16] in which the EEG signals are bandpass-filtered into some 
frequency bands and CSP features are extracted from each of these bands. Finally, a classification algorithm is used to classify the selected CSP features.The FBCSP algorithm used the typical estimation ofmultivariate covariance matrices from the EEG signals for a filter bank of CSP. Usually EEG signals are contaminated with artifacts or different types of noise sources. Due to the contamination the normal pattern of the majority of the EEG data are differed [17]. In the case of large amount of contamination, the multivariate covariance estimates typically varies significantly from the estimate without the contamination [17]. Therefore, the FBCSP algorithm is sensitive to artifacts in the trainingdata [14].

Arobust filter bank commonspatial pattern (RFBCSP) algorithm was proposed [18] where theminimum covariance determinant (MCD) estimator is used to estimate the covariance matrices. Likewise, to estimate the variance of the projected EEG signals the median absolute deviation (MAD) is used. The classification performance of the RFBCSP is better in some specific subjects but the overall results are not statistically significant.

In this paper, a novel approach is proposed for EEG signal classification in motor imagery-based BCI implementation. The proposed approach is subdivided into three stages. The EEG signal is first divided into multiple frequency bands using bandpass filter considering to capture the relevant brain rhythmic components, the CSP features are extracted from each of these frequency bands. A LDA based classification algorithm is used to classify the specific subband using the CSP features. The overall EEG classification is achieved by selecting the maximum LDA classification scores over that of the subbands.

The paper is organized as follows- Section 2 discusses a feature extraction technique called with CSP, the basics of LDA technique is explained in section 3 , the section 4 contains the description of the proposed method, the experimental results are illustrated in section 5 and the section 6 include discussion and some concluding remarks.

\section{Feature Extraction with CSP}

Common Spatial Pattern (CSP) is a feature extraction technique used in signal processing for separating a multivariate signal into additive sub-components.The technique used to design spatial filters such that the variance of the filtered data from one class is maximized while the variance of the filtered data from the other class is minimized. Thus, the resulting feature vectors increase the discriminability between the two classes by means of minimize the intra class variance and maximize the inter class variance [19]. This property builds CSP as one of the most effective spatial filters for EEG signal processing. The method of CSP was first introduced to EEG analysis for detection of abnormal EEG [20] and effectively applied on movement-related EEG for the classification purpose $[8,10]$. The target of the CSP is to project the multichannel EEG data into low dimensional spatial subspace with a projection matrix using linear transformation [21].

For details explanation of the CSP algorithm, assume the original EEG data matrix $E_{k}^{i}$ from trial $i$ for class $k$. The dimension of each $E_{k}^{i}$ is $D \times T$, where $D$ is the number of channels and $T$ is the number of samples per channel. For the explanation, the EEG data of a single trial $(i=1)$ is represented as $E_{k \in(h, f)}$ where $h$ denotes hand and $f$ denotesfoot movement. The normalized spatial covariance of the EEG for hand movement, $C_{h}$ and for the foot movement, $C_{f}$ can be calculated as:

$$
C_{h}=\frac{E_{h} E_{h}^{\prime}}{\operatorname{tr}\left(E_{h} E_{h}^{\prime}\right)}, \quad C_{f}=\frac{E_{f} E_{f}^{\prime}}{\operatorname{tr}\left(E_{f} E_{f}^{\prime}\right)}
$$

where $E_{h}$ and $E_{f}$ represent the original EEG matrices for hand and foot movement respectively, $(\cdot)^{\prime}$ is the transpose operator and $\operatorname{tr}(\cdot)$ represents the sum of the diagonal elements of any given matrix. The composite spatial covariance, $C$ is the sum of the averaged normalized spatial covariance $\bar{C}_{h}$ and $\bar{C}_{f}$. The $\bar{C}_{h}$ and $\bar{C}_{f}$ are estimated by averaging over all the trials of each class. The composite spatial covariance, $C$ is calculated as

$$
C=\bar{C}_{h}+\bar{C}_{f}=\Sigma \lambda \Sigma^{\prime}
$$

where $\sum$ is the matrix of Eigenvectorsand $\lambda$ is the diagonal matrix of Eigenvalues.The averaged normalized spatial covariance $\bar{C}_{h}$ and $\bar{C}_{f}$ are transformed as

$$
J_{h}=X \bar{C}_{h} X^{\prime} \text { and } J_{f}=X \bar{C}_{f} X^{\prime}(3)
$$

where $X=\Sigma^{\prime} / \sqrt{\lambda}$ is the whitening transformation matrix. $J_{h}$ and $J_{f}$ share common eigenvectors and the sum of corresponding eigenvalues for the two matrices will always be one. If $J_{h}=Y \Lambda_{h} Y^{\prime}$ and $J_{f}=Y \Lambda_{f} Y^{\prime}$ then $\Lambda_{h}+\Lambda_{f}=I$, where $I$ is the identity matrix. Since the sum of two corresponding eigenvalues is always one, a high eigenvalue for $J_{h}$ means that a high variance for EEG in hand movement and a low variance for the EEG in 
foot movement (low eigenvalue for $J_{f}$ ) and vice versa. The classification operation is done based on this property. The projection of whitened EEG onto the eigenvectors $Y$ corresponding to the largest $\Lambda_{h}$ and $\Lambda_{f}$ will give feature vectors that significantly enhance the discrimination ability.

The goal of the CSP is to find $B$ spatial filters to create a projection matrix $W$ of dimension $N \times B$ (each column is a spatial filter). The projection matrix $W$ is represented as

$$
W=Y^{\prime} X
$$

The projection matrix $W$ linearly transforms the original EEG into uncorrelated components according to:

$$
Z=W E \quad(5)
$$

The original EEG, $E$ can be reconstructed by $E=W^{-1} Z$ where $W^{-1}$ is the inverse matrix of $W$. The columns of $W^{-1}$ are spatial patterns that describe the variance of the EEG. The first and last columns contain the most discriminatory spatial patterns that explain the high variance of one class and the low variance of the other.

\section{LDA Based Classification}

Linear discriminant analysis (LDA), also known as Fisher's linear discriminant analysis is a technique used to find a linear combination of features that separates two or more classes of data. It is typically used as a dimensionality reduction step before classification [22]. It reduces dimensionality but at the same time preserves as much of the class discriminatory information as possible. The goal of the LDA is to use a separatinghyperplane that maximally separate the data representing the different classes. The hyperplane is found by selecting the projection, where the same classes are projected very close to each other and the distance between two classes means is as maximum as possible [23].Let as assume that we have $K$ classes, each containing $N$ observations $x_{i}$. The within-class scatter, $\widetilde{S}_{w}$ for all $K$ classes can be calculated as:

$$
\tilde{S}_{w}=\sum_{k=1}^{K} f_{k} S_{w}^{k}(6)
$$

where the within-class covariance matrix $S_{w}^{k}$ and the fraction of data $f_{k}$ are calculated according to the following formulas:

$$
\begin{gathered}
S_{w}^{k}=\sum_{i=1}^{N_{k}}\left(x_{i}^{k}-\mu^{k}\right)\left(x_{i}^{k}-\mu^{k}\right)^{T} \\
f_{k}=\frac{N_{k}}{\sum_{j=1}^{K} N_{j}}(8)
\end{gathered}
$$

where $N_{k}$ is the number of observations of $k^{\text {th }}$ classand $\mu^{k}$ indicates mean of the all observations $x_{i}$ for $k^{\text {th }}$ class. The between class scatter $\widetilde{S}_{b}$ for all $K$ classes is calculated as:

$$
\tilde{S}_{b}=\sum_{k=1}^{K} f_{k} S_{b}^{k}(9)
$$

where the between class covariance matrix, $S_{b}^{k}$ can be estimated as

$$
S_{b}^{k}=\sum_{k=1}^{K}\left(\mu^{k}-\mu\right)\left(\mu^{k}-\mu\right)^{T}(10)
$$

Where $\mu$ indicates the mean of the all observations $x_{i}$ for all classes. The main objective of LDA is to find a projection matrix that maximizes the ratio of the determinantof $\tilde{S}_{b}$ to the determinant of $\tilde{S}_{w}$. The projections that providing the best class separation are eigenvectors with the highest eigenvalues of matrix $M[22]$ :

$$
M=\frac{\tilde{S}_{b}}{\tilde{S}_{w}}(11)
$$

Since thematrix $M$ is asymmetric, the calculation of eigenvectors can be difficult. This difficulty can be minimized by using generalized eigenvalue problem [20]. Now, the aim of the LDA is to seek $(K-1)$ projections $\left[y_{1}, y_{2}, y_{3}, \ldots, y_{K-1}\right]$ by means of $(K-1)$ projection vectors. The transformed data set $y$ is obtained as a linear combination of all input features $x$ with weights $W$.

$$
y=x^{T} W(12)
$$

where $W=\left[w_{1}, w_{2}, w_{3}, \ldots, w_{H}\right]$ is a matrix form with the $H$ eigenvectors of matrix $M$ associated with the highest eigenvalues. The LDA reduces the original feature space dimension to $H$.The LDA performs well when the discriminatory information of data depends on the mean of the data. But it does not work for the variance depended discriminatory informative data. Also, the performance of the LDA is not good for nonlinear classification. 


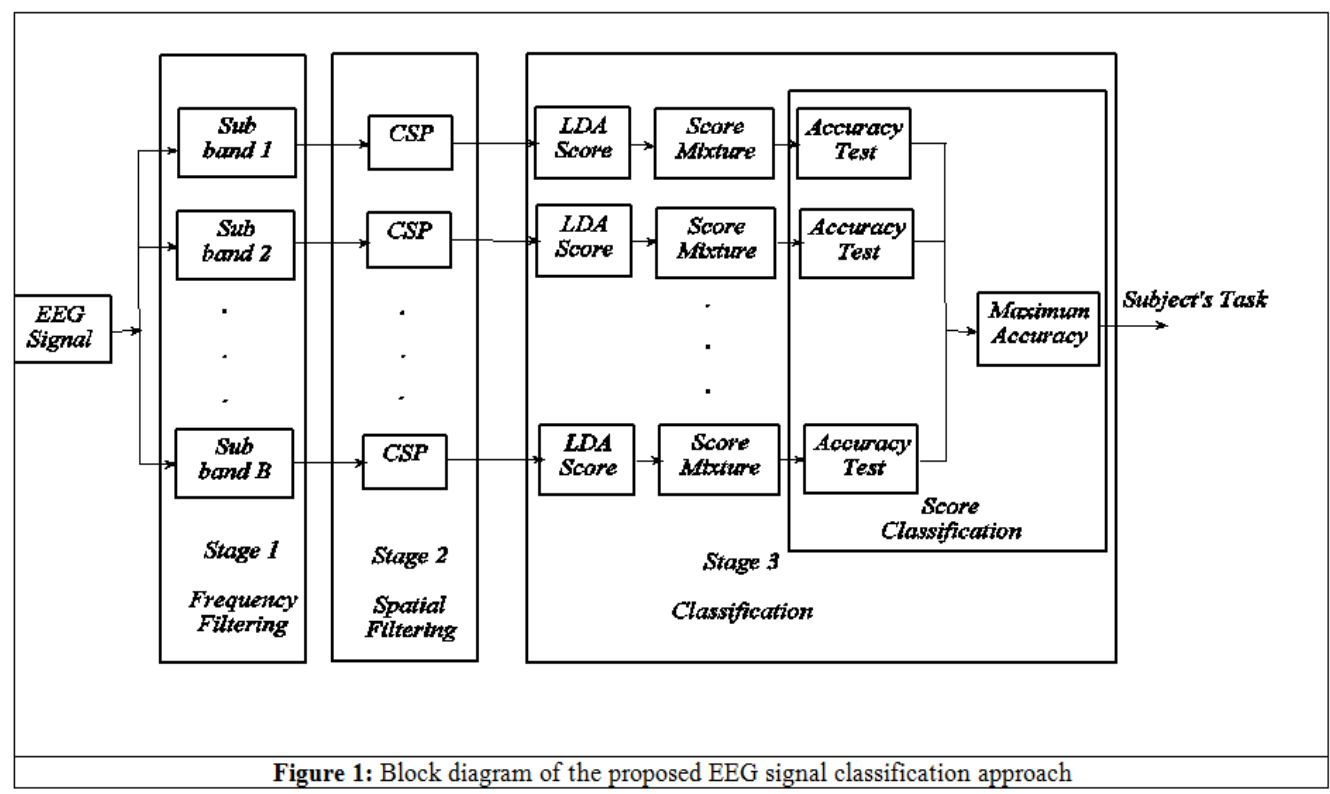

\section{Proposed Method}

The proposed EEG signal classification approach is illustrated in Fig. 1.This approach is subdivided into three stages for EEG signal processing and machine learning. In the first stage, the EEG signal frequency is filtered into multiple pass bands using bandpass filter. In the second stage, CSP features are extracted from each of these frequency bands. In the third stage, the classification operation is performed by finding LDA scores, blending and classifying the scores. A detail of each stage is described in below.

Subbanddecomposition: the first stage filters the EEG signal into multiple frequency passbands. The digital Butterworth bandpass filter is used to filter the EEG signal. Here, the most dominating rhythmic components alpha and beta $(8-32 \mathrm{~Hz})$ are selected. A total of six bandpass filters $8-12 \mathrm{~Hz}, 12-16 \mathrm{~Hz}, 16-20 \mathrm{~Hz}, 20-$ $24 \mathrm{~Hz}, 24-28 \mathrm{~Hz}$ and $28-32 \mathrm{~Hz}$ are used. The filtered sixsubbands are used individually for the classification.

Spatial filtering: The CSP algorithm is greatly successful in calculating spatial filters.In this stage, the CSP algorithm is used to perform the spatial filtering operation. The spatial filtering is done using the CSP algorithm by linearly transforming the EEG measurements using equation (5). The spatial filter thus produces reduced features for the particular frequency range of each of the subbands. The computed CSP features with optimal variances are used for discriminating the two classes ofEEG signals.

Classification:In the third stage, classification algorithm is implemented with LDA to classify the EEG trials using selected CSP features. Each subband feature is passed separately through the classifier. To validate the classification, $P \times Q$-foldcross validation is used. Here, $Q$ is the number of foldsfor cross validation. The data set is divided into $Q$ subsets and it repeats $Q$ times. Each time, one of the $Q$ subsets is used as the test set and the other $Q$-1subsets are put together to form a training set. The $Q$-fold cross validation is repeated $P$ times.At the first step of the classification, the LDA classifier produced $n$ LDA scores $\Phi_{i}(i=1,2, \ldots, Q)$ for every fold cross validation wheren is the total number of trials divided by the number of fold cross validation $Q$. During the score mixture, the LDA scores are mixed up by averaging the scores obtained from $Q$-fold cross validation. The score mixture of $Q$-fold cross validation is calculated as

$$
\Psi^{n}=\frac{1}{Q} \sum_{i=1}^{Q} \Phi_{i}(13)
$$

where, $\Psi^{n}$ denotes the mixture of $n$ LDA scores. The mixed LDA scores are converted to predicted classes. After $Q$-fold cross validation, accuracyistested comparing the predicted classes with the test set. The accuracy $\Gamma_{j \times Q}$ $(j=1,2, \ldots, P)$ is determined for $P$ times of validation. After computing $P \times Q$-fold cross validation, the classification scoreof individual subbandis estimated by averaging the accuracy of all $P$-fold cross validation as:

$$
\mathrm{A}_{b}=\frac{1}{P} \sum_{j=1}^{P} \Gamma_{j \times Q}
$$

where, $\mathrm{A}_{b}$ is the classification accuracy for subband $b(b=1,2, \ldots, B)$. Finally, the overall classification accuracy in of the EEG signal iscalculated by 


$$
C_{\text {accuracy }}=\underset{b}{\arg \max }\left(\mathrm{A}_{b}\right)(15)
$$

It is the maximum classification accuracy amongall ofthe subbands. The classification method is summarized as:

1) The LDA based classifier is implemented with CSP features of each subband

2) $P \times Q$-foldcross validation is used to validate the classification performance

3) The classifier generates LDA scores for each of $Q$-fold cross validation

4) The score mixture operation is done by averaging the scores obtained for all $Q$-fold with to equation (13)

5) The class prediction is performed using the mixed scores obtained from $Q$-fold cross validation for individual subband. The accuracy is tested by comparing the predicted classes with the test set.

6) Step 3 to 5 is repeated $P$ times and the classification score for each subband is calculated by averaging the score for all $P$-fold cross validation according to equation (14)

7) Finally, the maximum classification score among all thesubbands is the classification accuracy of the analysing EEG signal

\section{Experimental Results}

The performance of the proposed method is assessed by classifying the EEG of imagined movement. The proposed approach is applied to the publicly available BCI competition dataset. A filter bank is used to decompose the multichannel EEG signals into a desired number of subbands to capture alpha and beta rhythmic components $(8-32 \mathrm{~Hz})$. The filter bank comprises six bandpass filters with frequency limits $8-12 \mathrm{~Hz}, 12-16 \mathrm{~Hz}$, $16-20 \mathrm{~Hz}, 20-24 \mathrm{~Hz}, 24-28 \mathrm{~Hz}$ and $28-32 \mathrm{~Hz}$. A fourth-order Butterworth filter is used to subband the EEG data.To extract features from the data, the CSP algorithm with $m=2$ (pairs of CSP features)is used in this experiment.

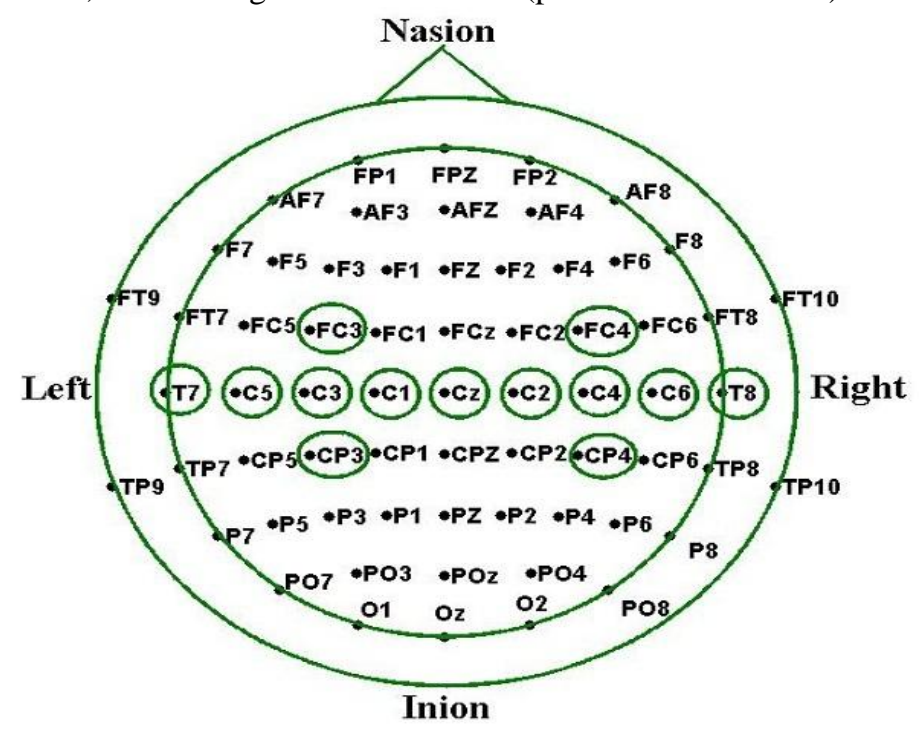

Figure 2:The electrodes map of 10/20 EEG systemstandardized by the American EEG society. The circled electrodes are considered for the dataset used in this experiment.

Dataset: To evaluate the performance of the proposed method, the dataset IVa from the publicly available BCI competition III 2005 [25] is used in this experiment. This dataset contains data from the four initial sessions without feedback. The dataset is recorded from five healthy subjects (labelled ' $a a$ ', ' $a l$ ', ' $a v$ ', ' $a w$ ', ' $a y$ ') who performed right hand and right foot movement imagination [26]. The data for each subject comprises 280 trials from 118 EEG channels and 140 trials in each class. The visual cues at each trial last for 3.5 seconds. The sampling rate of the data is $100 \mathrm{~Hz}$. In this experiment, the data between 0.5 seconds and 2.5 seconds from the visual cue (i.e. 200 time points at each trial) is extracted.

Channel selection: The motor imagery response of brain is more active in its central part [27]. In this experiment, out of the $118 \mathrm{EEG}$ channels, from the central area 13 are selected for classification. The selected EEG channels are 'FC3', 'FC4', 'Cz', 'C1', 'C2', 'C3', 'C4', 'C5', 'C6', 'T7', 'T8', 'CP3', and 'CP4'. The spatial distribution of the channels on the scalp in 10/20 EEG system is illustratedin Fig. 2. 

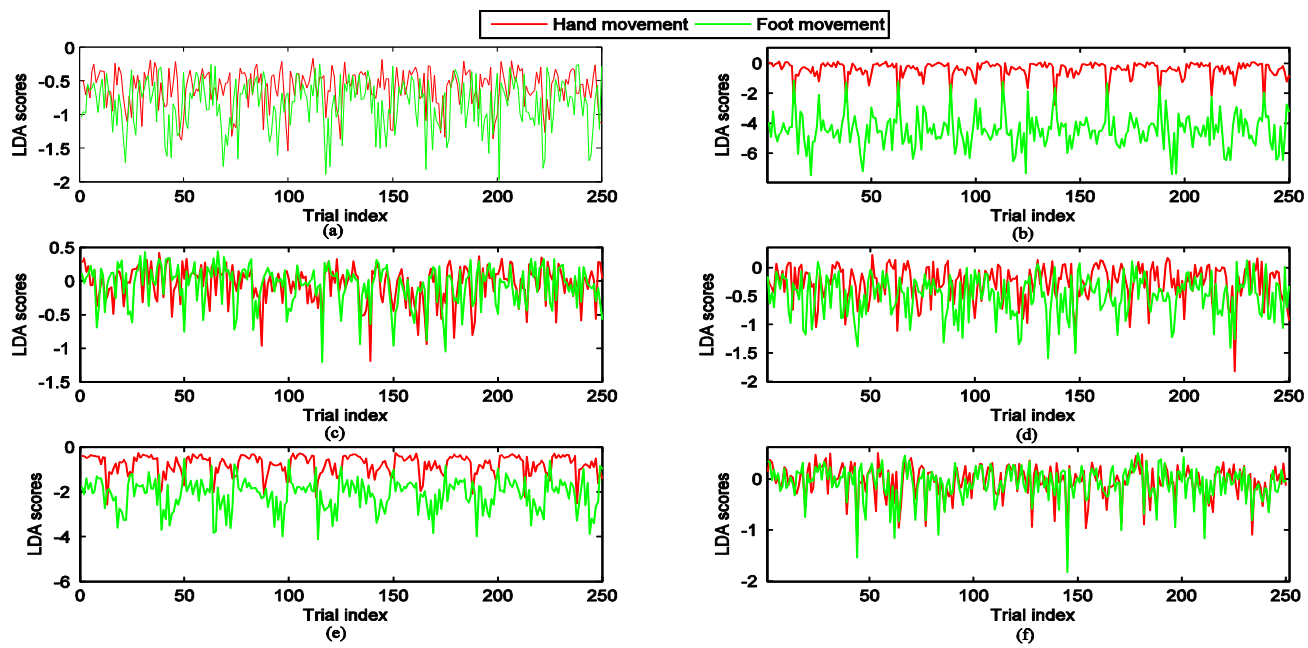

Figure 3: LDA scores as a function of trial index of subject ' $\boldsymbol{a} \boldsymbol{a}$ ' for different subbands: (a) $8-12 \mathrm{~Hz}$, (b) $12-$ $16 \mathrm{~Hz}$, (c) $16-20 \mathrm{~Hz}$,(d) $20-24 \mathrm{~Hz}$, (e) $24-28 \mathrm{~Hz}$ and (f) $28-32 \mathrm{~Hz}$.
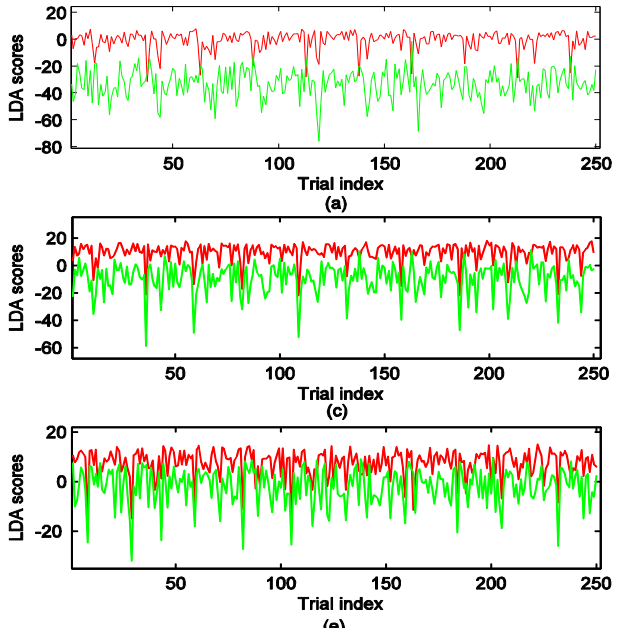
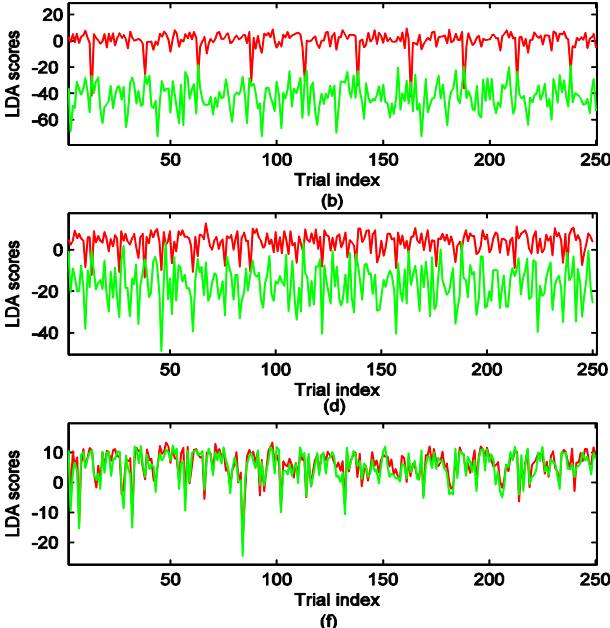

Figure 4: LDA scores as a function of trial index of subject ' $a l$ ' for different subbands: (a) $8-12 \mathrm{~Hz}$, (b) $12-16 \mathrm{~Hz}$, (c) $16-20 \mathrm{~Hz}$, (d) $20-24 \mathrm{~Hz}$, (e) $24-28 \mathrm{~Hz}$ and (f) $28-32 \mathrm{~Hz}$.
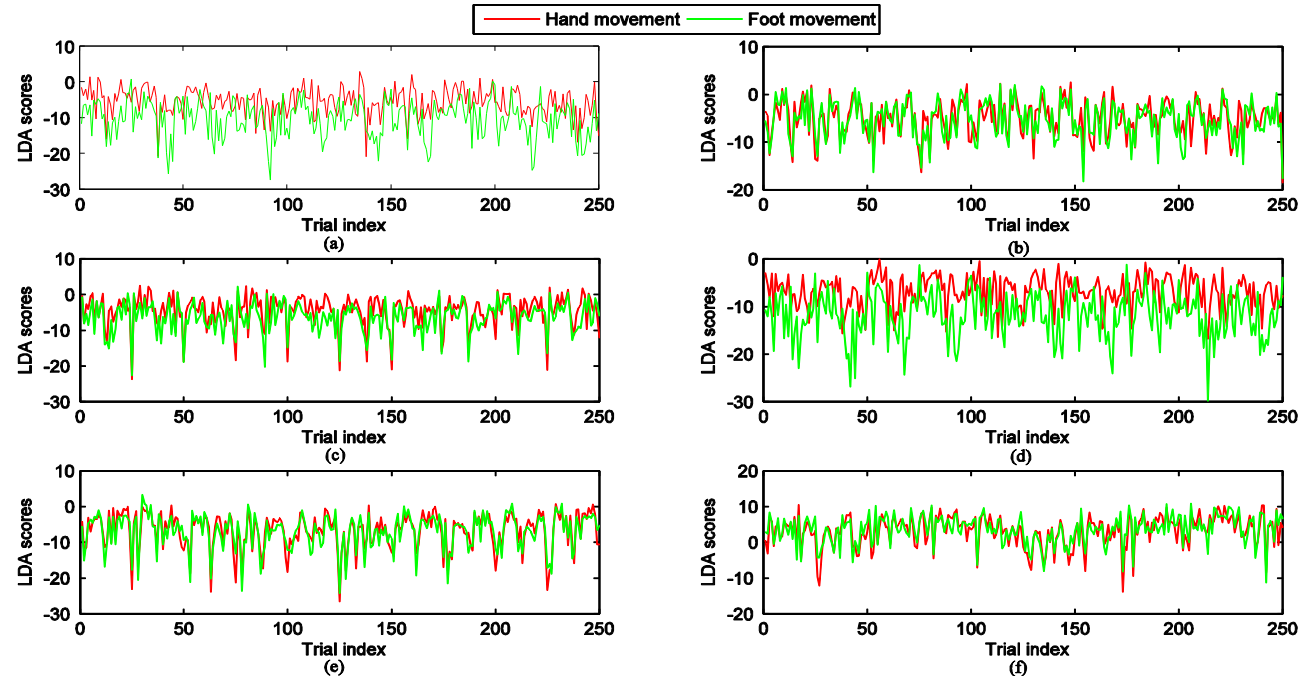

Figure 5: LDA scores as a function of trial index of subject ' $\boldsymbol{a} \boldsymbol{v}$ ' for different subbands: (a) $8-12 \mathrm{~Hz}$, (b) $12-$ $16 \mathrm{~Hz}$, (c) $16-20 \mathrm{~Hz}$, (d) $20-24 \mathrm{~Hz}$, (e) $24-28 \mathrm{~Hz}$ and (f) $28-32 \mathrm{~Hz}$. 

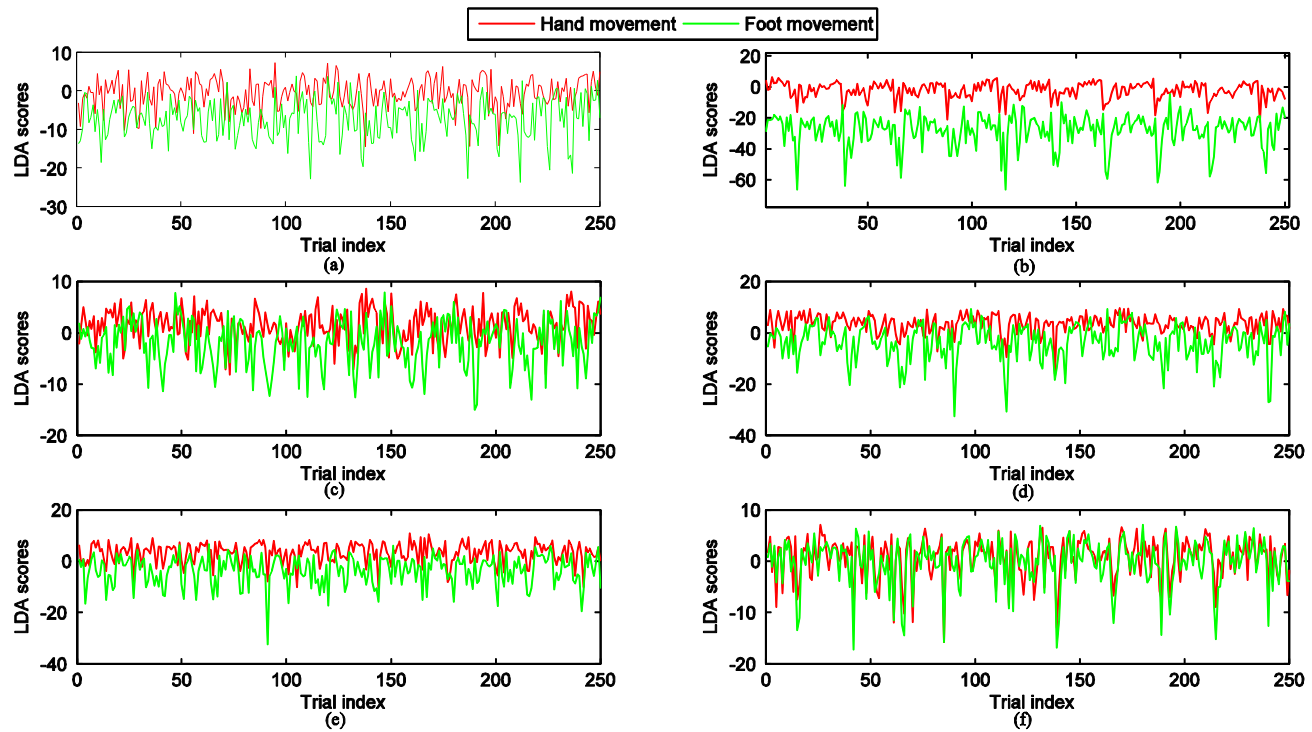

Figure 6:LDA scores as a function of trial index of subject ' $\boldsymbol{a} \boldsymbol{w}$ ' for different subbands: (a) 8-12Hz, (b) 12-16Hz, (c) $16-20 \mathrm{~Hz}$, (d) $20-24 \mathrm{~Hz}$, (e) $24-28 \mathrm{~Hz}$ and (f) $28-32 \mathrm{~Hz}$.
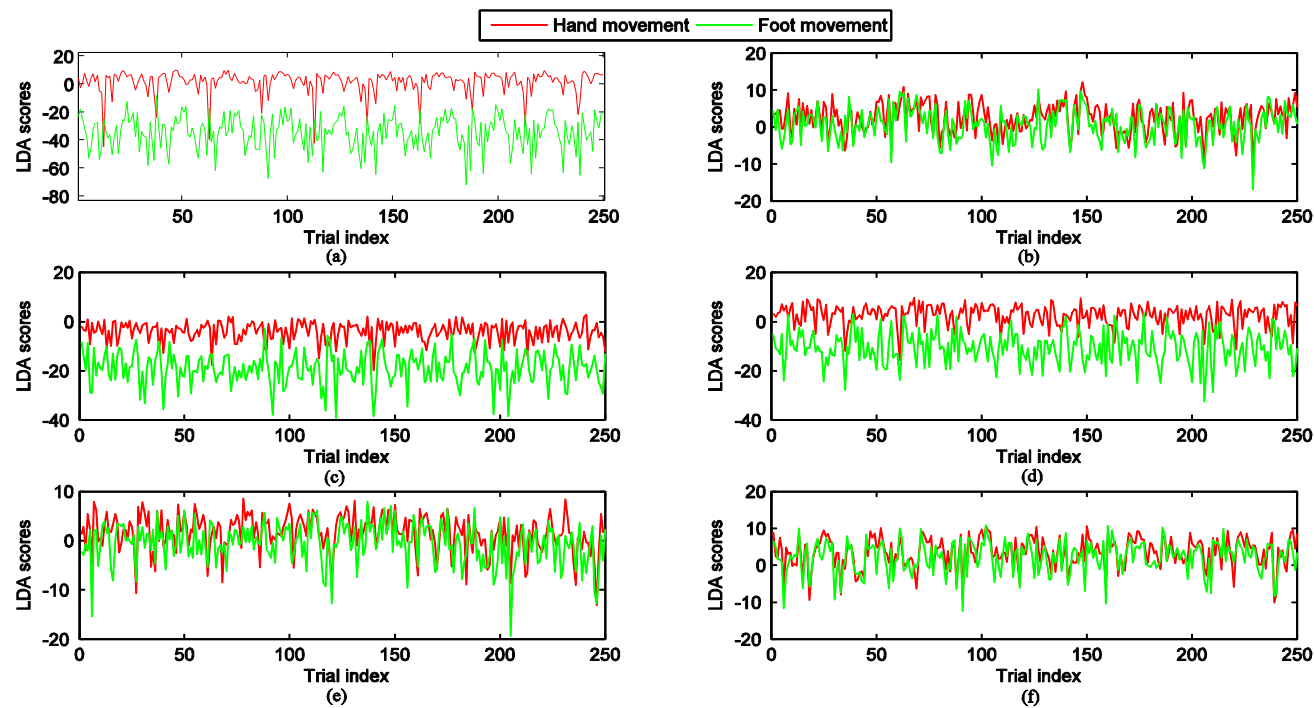

Figure 7:LDA scores as a function of trial index of subject 'ay' for different subbands: (a) $8-12 \mathrm{~Hz}$, (b) $12-16 \mathrm{~Hz}$, (c) $16-20 \mathrm{~Hz}$, (d) $20-24 \mathrm{~Hz}$, (e) $24-28 \mathrm{~Hz}$ and (f) $28-32 \mathrm{~Hz}$.

Fig. 3 to Fig. 7 show the LDA scores graphs for the five subjects 'aa', 'al', 'av', 'aw' and 'ay' respectively. The individual panel of any Figure represents the LDA scores for each of six subband $(8-12 \mathrm{~Hz}, 12-16 \mathrm{~Hz}, 16-20 \mathrm{~Hz}$, $20-24 \mathrm{~Hz}, 24-28 \mathrm{~Hz}$ and $28-32 \mathrm{~Hz}$ ) for both classes (right hand and right foot movement imagination). It is observed that the LDA scores for hand movement are greater than that of the foot movement. Such discriminative features enhance the classification accuracy of the proposed method. The topographical brain maps for subband 1 and subband 2 during imaginary right hand and right foot movement for the five subjects ('aa', 'al', ' $a v$ ', ' $a w$ ', ' $a y$ ') are shown in Fig. 8. The letter ' $\mathrm{L}$ ' and ' $\mathrm{R}$ ' indicates left and right hemisphere respectively. The most significant CSP of the two subbands are used for the topographical brain maps. The first and second trace (Fig. 8) show the topographical brain maps of the subband 1 for imaginary right hand and right foot movement respectively. The topographical brain maps of subband 2 for the imaginary right hand and right foot are shown in third and fourth trace (Fig. 8) respectively. It is noticed that the electrodes of right hemisphere are more activate (with higher energy) for right hand movement imagery action. With both subbands $(8-12 \mathrm{~Hz}$ and $12-16 \mathrm{~Hz}$ ), the front-central region of right hemisphere illustrate more energy that express the guess of imaginary right hand movement. On the other hand, the electrodes of left hemisphere are moreactivate for right foot action as shown in the second and fourth traces of Fig. 8. In this case the parietal-occipital region of the left hemisphere is more active with higher energy that illustrating the imaginary right foot movement action. 


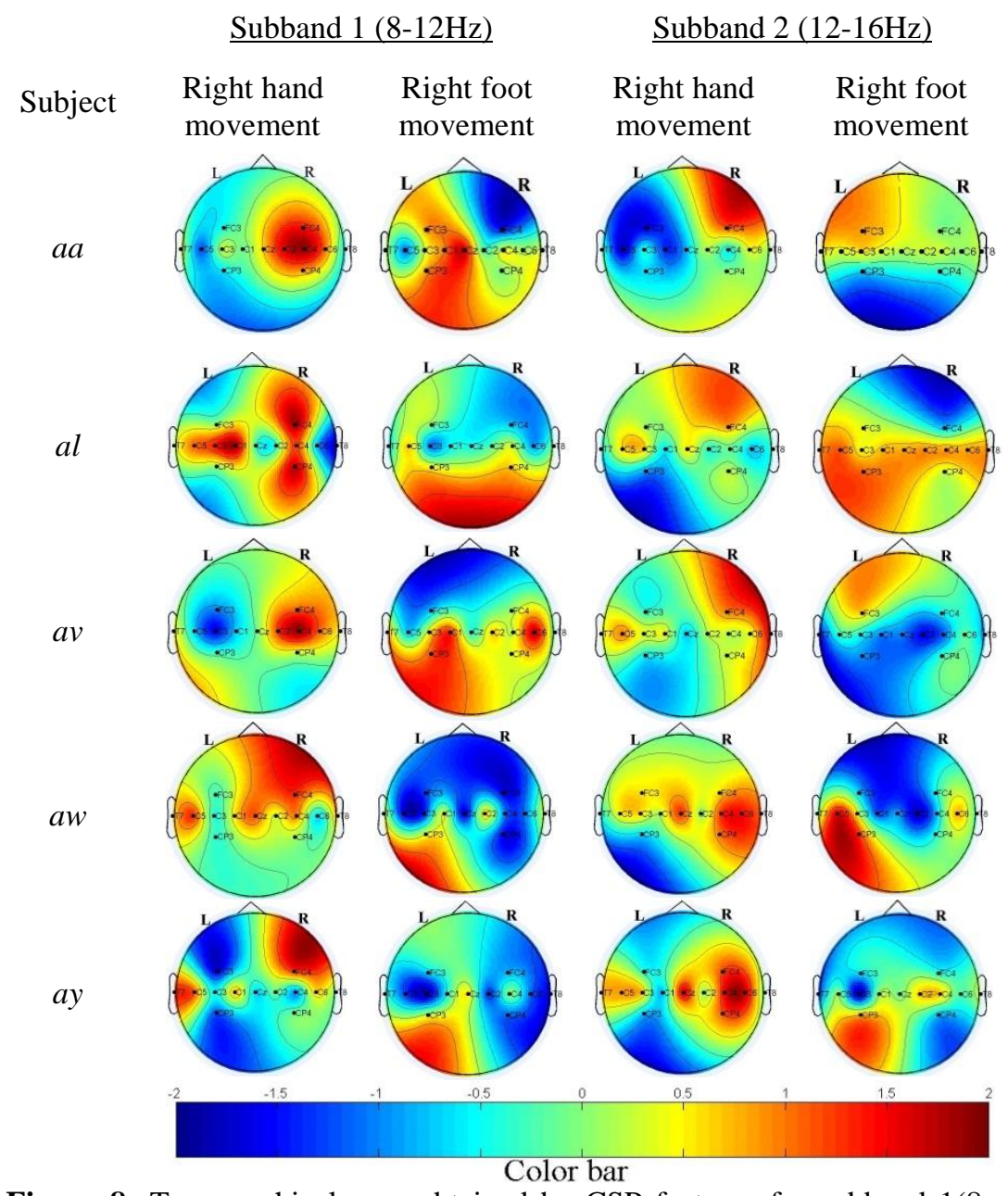

Figure 8: Topographical map obtained by CSP features for subband 1(8$12 \mathrm{~Hz})$ and subband $2(12-16 \mathrm{~Hz})$ of five subjects; first trace: right hand, second trace: right foot movement of subband 1; third trace: right hand, fourth trace: right foot movement of subband 2.

Table I: Classification accuracy (\%)

\begin{tabular}{|c|c|c|c|c|c|c|}
\hline \multirow{2}{*}{ Method } & \multicolumn{5}{|c|}{ Subject } & \multirow{2}{*}{ Mean \pm SD } \\
\cline { 2 - 6 } & $a a$ & $a l$ & $a v$ & $a w$ & $a y$ & \\
\hline CSP1[28] & 71.30 & 88.40 & 48.60 & 89.90 & 79.90 & $75.60 \pm 15.06$ \\
\hline CSP2[28] & 65.30 & 90.20 & 63.70 & 80.30 & 87.30 & $77.40 \pm 10.99$ \\
\hline EMD-CSP[28] & 68.40 & 89.60 & 64.10 & 82.50 & 86.90 & $78.30 \pm 10.19$ \\
\hline MEMD1-CSP[28] & 68.80 & 90.00 & 68.80 & 76.30 & 87.50 & $78.30 \pm 9.01$ \\
\hline MEMD2-CSP[28] $^{2}[60.30$ & 82.90 & 55.30 & 60.70 & 74.00 & $66.60 \pm 10.22$ \\
\hline FBCSP $_{\mathrm{w}}[16]$ & 93.30 & $\mathbf{9 8 . 5 0}$ & 66.80 & 93.80 & 93.60 & $89.20 \pm 11.36$ \\
\hline FBCSP $_{\mathrm{f}}[16]$ & 86.00 & 97.90 & 76.80 & 96.80 & 94.00 & $90.3 \pm 7.93$ \\
\hline Proposed MbCSP & $\mathbf{9 5 . 4 0}$ & $\mathbf{9 8 . 4 0}$ & $\mathbf{8 5 . 2 0}$ & $\mathbf{9 8 . 2 0}$ & $\mathbf{9 6 . 4 0}$ & $\mathbf{9 4 . 7 2 \pm 4 . 8 9}$ \\
\hline
\end{tabular}

Table I shows the classification accuracy with unbiased $10 \times 10$-fold cross validations. The performance of the proposed method is compared with that of the other methods (CSP, EMD-CSP and MEMD-CSP) proposed in [28] and methods $\left(\mathrm{FBCSP}_{\mathrm{w}}, \mathrm{FBCSP}_{\mathrm{f}}\right)$ proposed in [16].In CSP1, all of 118 EEG channels are used, whereas, selected 51-57 channels are used in CSP2. The univariate EMD is used to decompose the individual channels of EEG and some intrinsic mode functions (IMFs) are selected leading to use of CSP in EMD-CSP method. In MEMD1-CSP method, the proper IMFs are selected using heuristic method. The fractional Gaussian noise (fGn) is used as the reference signal in MEMD2-CSP to select the effective IMFs prior to apply CSP for feature selection. The wrapper-based feature selection technique (using classifier) is used FBCSP to implement FBCSP $_{w}$, whereas, filter-based algorithm is used for $\mathrm{FBCSP}_{\mathrm{f}}$ which is independent of classifier. It is observed 
that our proposed MbCSPmethod yields superior average classification accuracy (94.72 \pm 4.89$)$ than all the reported methods.

\section{Discussion and Conclusions}

A novel method to classify EEG of imagined right hand and right foot movement is introduced in this paper. The EEG is filtered into multiple subbands for the purpose of selecting an appropriate frequency bands corresponding to the potential rhythmic components to enhance the classification accuracy. The band-specific discriminative CSP features are then extracted. The CSP features of each subband are passed though LDA classifier. Traditionally, the LDA scores are generated and used to predict the class to measurethe accuracy. In the proposed MbCSP approach, the LDA scores are averaged over the cross validations. The average LDA scores are employed to predict the class and then accuracy is tested. There is a noticeable difference between LDA scoresof hand and foot actions (Fig. 3 to Fig. 7). For most of the subjects, the scores ofthe first subband(8$12 \mathrm{~Hz})$ and second subband $(12-16 \mathrm{~Hz})$ are more discriminative between the two classes compare to other subbands. However, for subject 'av'(in Fig. 5), the LDA scores of two actions for all subbands are very close to each other i.e. the two classes are not so much discriminative. That is why the relatively inferior classification accuracy is obtained for subject 'av'. The LDA scores are mixed up and the mixed scores are used in prediction. The classification accuracy of each subband is measured individually. The subband that contains the maximum discriminative features between classes produced maximum classification accuracy. The maximum accuracy is considered as the classification accuracy of the EEG signal.

In the frequency filtering stage of FBCSP,the signal of frequency limits $4-40 \mathrm{~Hz}$ is used for classification, whereas, the most dominant frequency bands alpha and beta $(8-32 \mathrm{~Hz})$ is used in the proposed method (MbCSP). In FBCSP, a feature selection algorithm is used to select a reduced size of CSP features through $\mathrm{FBCSP}_{\mathrm{w}}$ or $\mathrm{FBCSP}_{\mathrm{f}}$. The reduced CSP features are then used for classification. The FBCSP algorithm used the typical estimation ofmultivariate covariance matrices from the EEG signals. Usually EEG signals are contaminated with artifacts generated from different noise sources. In the case of large amount of contamination, the multivariate estimated covariance typically varies significantly from the estimate without contamination [17]. Therefore, the FBCSP algorithm is sensitive to artifact contamination of trainingdata [14].In the proposed method MbCSP, the CSP features are passed to generate LDA scores leading to the score mixture. The LDA scores are mixed up by averaging the scores obtained from the number offolds used in cross validation. The effect of contamination is minimized and the common dominant features appear as prominent during the score mixture implemented by averaging. Hence, the improved classification accuracy of the proposed method is achieved.

\section{Acknowledgements}

This research work supported by the Information and Communication Technology (ICT) division of the ministry of Post, Telecommunication and Information Technology, Bangladesh.

\section{References}

[1]. B. Rebsamen, E. Burdet, C. Guan, H. Zhang, C. L. Teo, Q.Zeng, C.Laugier, and M. H. Ang Jr., "Controlling a Wheelchair Indoors UsingThought," IEEE IntelligentSystems, vol. 22, no. 2, pp. 18-24, 2007.

[2]. N. Birbaumer, "Brain-computer-interface research: Coming of age," Clin. Neurophysiol., vol. 117, no. 3, pp. 479-483, 2006.

[3]. J. R. Wolpaw, N. Birbaumer, D. J. McFarland, G. Pfurtscheller and T. M. Vaughan, "Brain-computer interfaces for communication andcontrol,"Clin. Neurophys., vol. 113, pp. 767-791, 2002.

[4]. E. Niredermeyer and F. Lopes da Silva, Electroencephalography: BasicPrinciples, Clinical Applications and Related Fields, 5th ed., Baltimore,MD, USA: Lippincott Williams Wilkins, 2004.

[5]. G. Pfurtscheller, C. Neuper, D. Flotzinger, andM.Pregenzer, "EEG baseddiscrimination between imagination of right and left hand movement,"Electroencephalography Clin. Neurophysiol., vol. 103, pp. 642-651, Dec.1997.

[6]. V. Carmen, N. Kramer, B. Blankertz, and A. Schlogl, "Time domainparameters as a feature for EEG-based Brain-Computer Interface," NeuralNetw., vol. 22, pp. 1313-1319, Jul. 2009.

[7]. T. Wang, J. Deng, and B. He, "Classifying EEG-based motor imagerytasks by means of time-frequency synthesized spatial patterns," Clin. Neurophysiol.,vol. 115, pp. 1-7, Jan. 2004.

[8]. H. Ramoser, J. M"uller-Gerking, and G. Pfurtscheller, "Optimal spatial filtering of single trial EEG during imagined hand movement," IEEE Transactions on Rehabilitation Engineering, vol. 8, no. 4, pp. 441-447, 2000.

[9]. G. Dornhege, B. Blankertz, M. Krauledat, F. Losch, G. Curio, and K.- R. M"uller, "Optimizing spatio-temporal filters for improving Brain-Computer Interfacing," in Advances in Neural Inf. Proc. Systems (NIPS05), J. Platt, Ed., vol. 18, Vancouver, Canada, December 2005.

[10]. J. Müller-Gerking, G. Pfurtscheller, and H. Flyvbjerg, "Designing optimalspatial filters for single-trial EEG classification in a movementtask," Electroenc.Clin.Neurophys., pp. 787-798, 1999.

[11]. Q. Novi, C. Guan, T. H. Dat, and P. Xue, "Sub-band Common Spatial Pattern (SBCSP) for Brain-Computer Interface,"3rd InternationalIEEE/EMBS Conference on Neural Engineering, 2007.CNE '07, pp. 204-207, 2007.

[12]. G. Dornhege, B. Blankertz, M. Krauledat, F. Losch, G. Curio, and K. R. Muller, "Combined Optimization of Spatial and Temporal Filters for Improving Brain-Computer Interfacing," IEEE Trans. Biomed. Eng., vol. 53, no. 11, pp. 2274-2281, 2006.

[13]. S. Lemm, B. Blankertz, G. Curio, and K.-R. Muller, "Spatio-Spectral Filters for Improving the Classification of Single Trial EEG," IEEETrans. Biomed. Eng., vol. 52, no. 9, pp. 1541-1548, 2005.

[14]. R. Tomioka, G. Dornhege, G. Nolte, B. Blankertz, K. Aihara, and K.-R Müller, "Spectrally Weighted Common Spatial Pattern Algorithm for SingleTrialEEGClassification”,Mathematical Engineering Technical Reports,UniversityofTokyo, Tokyo, 2006. 
[15]. K. K. Ang, Z. Y. Chin, C. Wang, C. Guan and H. Zhang, "Filter bank common spatial pattern algorithm on BCI competition IV Datasets 2a and 2b," frontiers in NEUROSCIENCE, vol. 6, 2012.

[16]. K. K. Ang, Z. Y. Chin, H. Zhang, and C. Guan, "Filter Bank Common Spatial Pattern (FBCSP) in Brain-Computer Interface," in Proc.IJCNN'08, pp. 2391-2398, 2008.

[17]. M. Hubert, P. J. Rousseeuw, and S. Van Aelst, "High-Breakdown Robust Multivariate Methods," Stat. Sci., vol. 23, no. 1, pp. 92$119,2008$.

[18]. K. K. Ang, Z. Y. Chin, H. Zhang, and C. Guan, "'Robust Filter Bank Common Spatial Pattern (RFBCSP) in motor-imagery-based Brain-Computer Interface", 31st Annual International Conference of the IEEE EMBSMinneapolis, Minnesota, USA, pp. 578-581, September 2-6, 2009.

[19]. Rajesh P. N. Rao, "Brain-Computer Interfacing: An Introduction,” Cambridge University Press, USA, 2013.

[20]. Z. J. Koles, "The quantitative extraction and topographic mapping ofthe abnormal components in the clinical EEG," Electroencephalography and Clinical Neurophysiology,vol. 79, no. 6, pp. 440-447, 1991.

[21]. Y. Wang, S. Gao, X. Gao, "Common spatial pattern method for channel selection in motor imagery based brain computer interface," IEEE Engineering in Medicine and Biology, $27^{\text {th }}$ Annual Conference, pp. 5392-5395, 2005.

[22]. M. Kantardzic, “Data Mining: Concepts, Models, Methods, andAlgorithms ”, IEEE Press \& John Wiley, November 2002.

[23]. M.R.Hasan, M.I.Ibrahimy,S.M.A.Motakabber and S.Shahid, "Classification of Multichannel EEG Signal by Linear Discriminant Analysis," Progress in Systems Engineering: Proceedings of the Twenty-Third International Conference on Systems Engineering, Advances in Intelligent Systems and Computing 1089, pp. 279-282, 2015.

[24]. I. E. Gareiset. al., "Determination of an optimal training strategy for a BCIclassification task with LDA," Neural Engineering (NER), pp. $286-289,2011$.

[25]. B. Blankertz, "BCI Competition III", Fraunhofer FIRST.IDA,http://ida.first.fraunhofer.de/projects/bci/competition_iii,2005.

[26]. G. Dornhege, B. Blankertz, G. Curio, and K.-R. Muller, "Boosting bitrates in noninvasive EEG single-trial classifications by featurecombination and multiclass paradigms," IEEE Trans. Biomed. Eng.,vol. 51, no. 6, pp. 993-1002, 2004.

[27]. C. Neuper, G. R. M“uller-Putz, R. Scherer, and G. Pfurtscheller, "Motorimagery and EEG-based control of spelling devices and neuroprostheses,'Progress in Brain Research, vol. 159, pp. 393-409, 2006.

[28]. L. Zhang, C. Shang, H. Higashi, J. Cao, and T. Tanaka, "Common Spatial Pattern Using Multivariate EMD for EEG Classification,"Asia-Pacific Signal and Information Processing Association Annual Summit and Conference 2011, October 18-20, Xi'an China, 2011. 